\title{
The Application of Multiple Wireless Sensor Data Fusion Algorithm in Temperature Monitoring System
}

\author{
Yongliang Wang ${ }^{1}$, Hongdou Chen ${ }^{2}$, Qi Zhang ${ }^{3}$ and Huimin $\mathrm{Lv}^{1+}$ \\ ${ }^{1}$ Tianjin University of Technology and Education, Tianjin Key Laboratory of Information Sensing \& \\ Intelligent Control, Tianjin, China \\ ${ }^{2}$ Sanshui District Industrial Secondary School in Foshan City, Guangdong Province, China \\ ${ }^{3}$ Hangzhou Xiaoshan Technician College, Zhejiang Province, China
}

\begin{abstract}
In order to increase accuracy of temperature monitoring system in greenhouse, this paper proposed a system based on multi-wireless sensor data fusion algorithm. Firstly, the algorithm based on Dixon criterion eliminated a gross error data, which acquired by the wireless control terminal. Then calculated the arithmetic mean and sent it to the coordinator node, finally, the adaptive weighted fusion algorithm was used to conduct the greenhouse temperature and get the accurate greenhouse temperature. Through the experiments showed: compared with traditional method of average mean, this data fusion algorithm improved the sensor measurement accuracy, had smaller error and improved the system stability.
\end{abstract}

Keywords: wireless sensor; data fusion; adaptive weighted

\section{Introduction}

Greenhouse uses microelectronics technology and modern control technology, through the control system for data analysis and processing, controlling the greenhouse water pumps, fans, sun visor and other equipments, which creating the most suitable environment for the growth of vegetables. So as to achieve the purpose of regulating crop breeding cycle, increasing crop yield, crop quality and improving economic efficiency [1-2]. Greenhouse environment is a complex distributed parameter system with nonlinear timevarying, multi-variable strong correlation large delay. The change of one parameter will affect the change of multiple environmental parameters [3]. Nowadays, the greenhouse temperature monitoring system uses a single sensor for data collection, because the sensor will be interfered by various factors and its own accuracy, its monitoring results will be a certain deviation. In addition, because of the large greenhouse space, the environmental parameters of different positions are different, and the single sensor cannot accurately reflect the greenhouse environment [4]. To accurately grasp the greenhouse environmental parameters, multi-sensor coordination must be used to complete the monitoring tasks. Reasonable selection of multisensor data fusion algorithm and improve monitoring data accuracy has become one of the key tasks of greenhouse monitoring.

This paper presented a temperature monitoring system based on multi-sensor data fusion. Firstly, the anomaly data of each acquisition node was eliminated by using the Dixon criterion. Then processing of the obtained conformance measurement data with the method based on the mean fusion processing, and the fusion result was sent to the coordinator node. Finally, the fusion data of each acquisition node was adaptively weighted fusion according to the principle of optimal allocation of weights. Through the multipoint temperature measurement of the collected nodes and fusion processing the data of the coordinator node,

+ Corresponding author. Tel.: 13920217350.

E-mail address: 1vhm0403@163.com. 
the accuracy of the temperature in the greenhouse was calculated by reducing the data transmission while ensuring the accuracy of the data.

\section{System Design}

The system used CC2530 as the development platform to build a multi-point temperature sensor network based on the control terminal and a coordinator. Each control terminal was a data acquisition node, which connected eight temperature sensors of the same model, and the data acquisition node collected data according to the acquisition cycle instruction of the system. Due to the uneven distribution of temperature in greenhouse, the different control terminals obtained temperatures in different spatial positions, the gross errors were eliminated by the Dixon criterion, and the remaining data was processed by the arithmetic mean method, then transmitted the data to the coordinator terminal via radio frequency. The coordination device brought the real environment condition of each collection node area and used adaptive weighted fusion method for data fusion to obtain the accurate temperature of the greenhouse. The fusion scheme is as shown in Fig. 1.

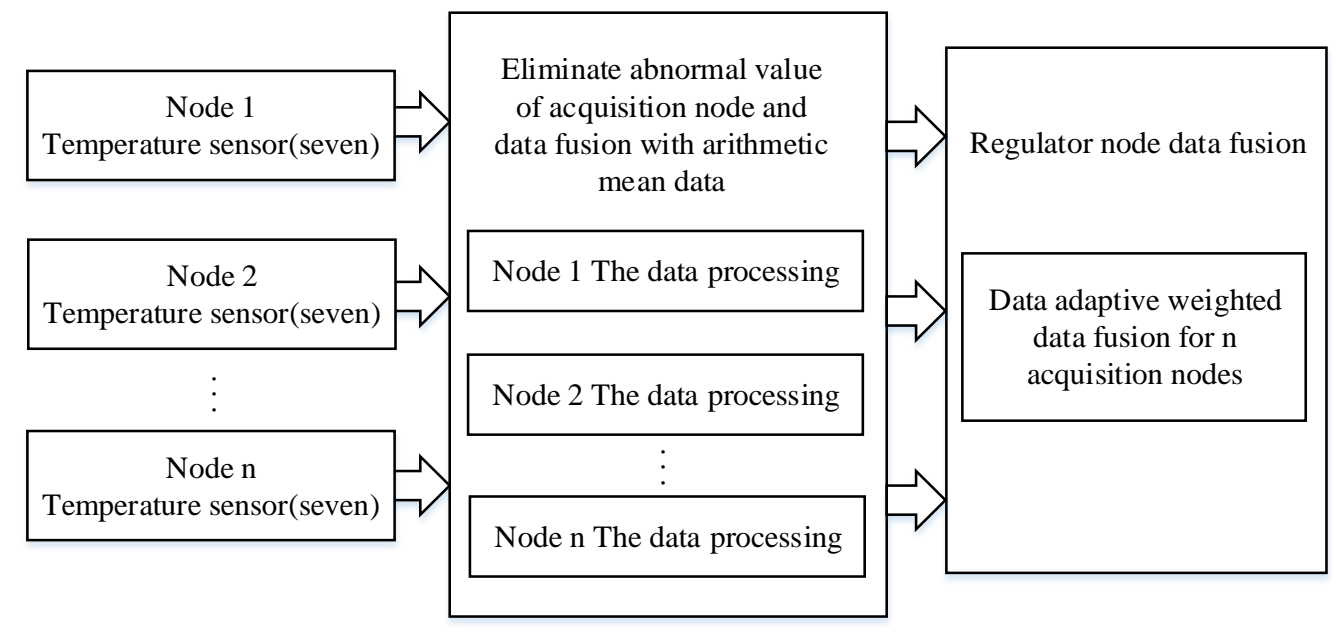

Fig. 1: Fusion scheme.

\section{Control Terminal Data Processing}

In a group of monitoring data, if there are individual values that differ greatly from other monitoring values, the obvious difference with other numerical data is referred to as the abnormal data, and the abnormal data reduces the credibility of the data which should be removed. There are four more commonly used criteria for monitoring the consistency of the monitoring values. The elimination method of gross error mainly includes Laiyite criterion, Lomnaofski norm, Grubbs criterion and Dixon criterion [5]. At present, there is no uniform criterion for the elimination of abnormal values. When the measurement data is few, the Dixon criterion can judge the coarse error quickly with less computation; When there is only one abnormal value in a set of measured values, it is optimal to use the Grubbs criterion; When a small amount of sample, the statistics critical coefficient of the Laiyite criterion is relatively large, it is difficult to find abnormal data in time and will be more harsh when using it; The statistical criticality coefficient of Lomnaofski criteria is too small and it is easy to get rid of the measurement value with only a large normal error [7]. In order to eliminate the larger error data and improve the accuracy, this paper uses the Dixon criterion to deal with the measured data.

Set the samples are $X_{1}, X_{2}, X_{3}, \cdots, X_{n}(n=3 \sim 7)$, and $X(1) \leq X(2) \leq X(3) \leq \cdots \leq X(n)$. When $X(i)$ obeys a normal distribution, the statistics are as follows:

$$
\begin{aligned}
& \gamma_{10}=\frac{x(n)-x(n-1)}{x(n)-x(1)} \\
& \gamma_{10}^{\prime}=\frac{x(2)-x(1)}{x(n)-x(1)}
\end{aligned}
$$


Take the significant level alpha as 0.01 , and the critical value $\mathrm{D}(\alpha, \mathrm{n})$ is obtained, if $\gamma_{10}>\gamma_{10}^{\prime}, \gamma_{10}>\mathrm{D}(\alpha, \mathrm{n}), \mathrm{X}(\mathrm{n})$ is abnormal data; If $\gamma_{10}<\gamma_{10}^{\prime}, \gamma_{10}^{\prime}>\mathrm{D}(\alpha, \mathrm{n}), \mathrm{X}(1)$ is abnormal data, or there is no abnormal data.

After removing the abnormal value, using the arithmetic mean method to integrate the remaining data, and the merged data is sent to the coordinator node. Each instruction node needs to send a set of data to extend the service life.

\section{Coordinator Node Data Fusion}

The data collected from each control terminal node was removed by the Dixon criterion, and get the consistency measurement data. These data from different nodes, and correspond to different weights. According to the principle of weight optimization, the weighted fusion algorithm is used to weighted fusion of each data, and the final fusion value is obtained [6]. The model of adaptive weighted fusion algorithm is as shown in Fig. 2.

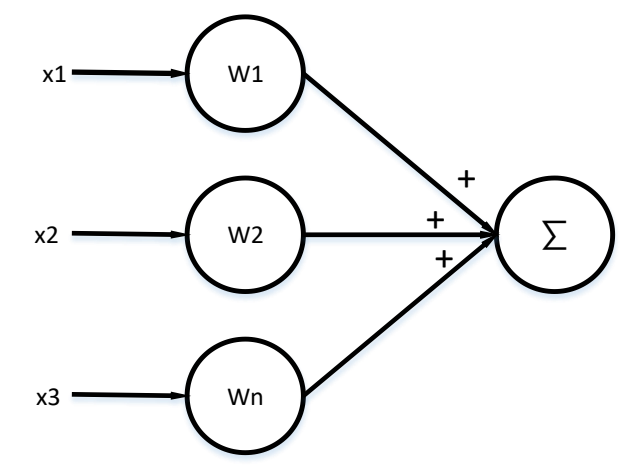

Fig. 2: Adaptive weighted average data fusion algorithm estimation model.

Let the variance of $\mathrm{n}$ sensors be $\sigma_{1}^{2}, \sigma_{2}^{2}, \cdots, \sigma_{n}^{2}$, the true value to be estimated is $X$, the measured values of each sensor are $X_{1}, X_{2}, \cdots, X_{n}$. They are independent of each other and are unbiased estimates of $X$, the weights are $W_{1}, W_{2}, \cdots, W_{n}$. Then the fusion value and the weighting factors are satisfied: $\hat{X}=\sum_{p=1}^{n} W_{p} X_{p}, \sum_{p=1}^{n} W_{p}=1$.

The total mean square error is $\sigma^{2}=E\left[(X-\hat{X})^{2}\right]$. Because the sensor data can be approximately independent of each other, there is $E\left\lfloor\left(X-X_{p}\right)\left(X-X_{q}\right)\right\rfloor=0(p=1,2, \ldots, n ; q=1,2, \ldots, n)$.

Therefore, the total mean square error can be written: $\sigma^{2}=W_{p}^{2} \sigma_{p}^{2}$. According to the multivariate function, the maximum value of the total mean square error is $W_{p}=\frac{1}{\sigma_{p}^{2} \sum_{i=1}^{n} \frac{1}{\sigma_{i}^{2}}(p=1,2, \ldots, n)}$.

\section{Test Analysis}

The test site was a greenhouse for vegetable planting in Tianjin, and the temperature acquisition data of the greenhouse in March 2017 was extracted, as shown in Table 1.

Table 1: Temperature data in greenhouse

\begin{tabular}{cccccc}
\hline Number & Node 1 & Node 2 & Node 3 & Node 4 & Node 5 \\
\hline 1 & 18.2 & 18.7 & 18.3 & 16.2 & 18.8 \\
2 & 17.9 & 18.5 & 18.1 & 16.1 & 18.7 \\
3 & 16.9 & 18.1 & 17.4 & 16.2 & 18.5 \\
4 & 17 & 18.2 & 17.2 & 16.8 & 19 \\
5 & 17.6 & 19 & 17.3 & 16.8 & 17.9 \\
6 & 17.5 & 18.4 & 17.9 & 16.6 & 18.4 \\
7 & 17.1 & 19.3 & 17.5 & 18.5 & 18.1 \\
\hline
\end{tabular}

Table 1 is the data collected by the onetime acquisition instruction, which contains the data of 5 sampling nodes, and each collection node has 7 temperature sensors of the same type. Take the node 3 as an 
example, the acquisition data of node 3 is processed with abnormal data. A series of temperature data in node 4 are sorted from small to big: 16.1, 16.2, 16.2, 16.6, 16.8, 16.8, 18.5. The calculated statistics $\gamma_{10}=0.71$, $\gamma_{10}^{\prime}=0.04$. And $D(0.01,6)=0.698, \gamma_{10}>\gamma_{10}^{\prime}, \gamma_{10}>\mathrm{D}(0.01,7)$, so 18.5 is an outlier.

Repeat this process to eliminate abnormal data of node 1-5, then compute the arithmetic mean, send it to the coordinator node, and alculate the variance and data weight of each node. The data is shown in Table 2.

Table 2: Coordinate Node Data

\begin{tabular}{cccc}
\hline Number & Data & Variance & Weight \\
\hline 1 & 17.46 & 0.20 & 0.08 \\
2 & 18.60 & 0.16 & 0.13 \\
3 & 17.67 & 0.16 & 0.14 \\
4 & 16.45 & 0.09 & 0.46 \\
5 & 18.40 & 0.14 & 0.18 \\
\hline
\end{tabular}

The adaptive weighted fusion estimation value: $\hat{X}=\sum_{p=1}^{n} W_{p} X_{p}=17.34^{\circ} \mathrm{C}$

Through continuous acquisition of temperature data for a period of time and compared with the arithmetic mean method.

Connection of temperature data within the continuous period, the data processing result was compared with that of the traditional arithmetic average method, and the result was as shown in Figure 3. The figure showed the fused data values were smooth, with high reliability, achieving the desired effect.

The results were processed by continuous acquisition of temperature data for a period of time and compared with the arithmetic mean method, the result was as shown in Figure 3.

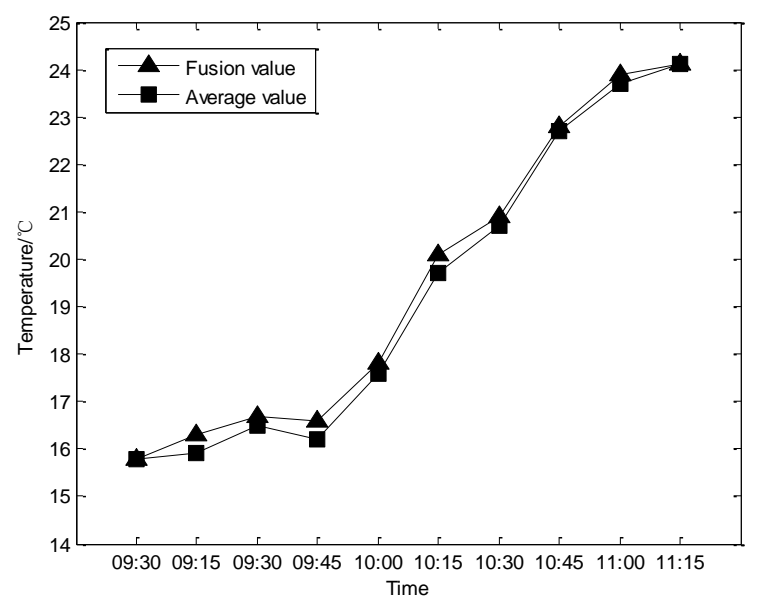

Fig. 3: Temperature fusion curve.

It can be seen from the above results, the fusion curve of this method is smooth and relatively small, which can reflect the changing conditions of greenhouse environment.

\section{Conclusion}

In this paper, we proposed a method to eliminate the abnormal data of acquisition node by Dixon criterion, then sent the arithmetic mean to the coordinator node, at last, the adaptive weighted fusion algorithm was used to conduct the greenhouse temperature and get the accurate greenhouse temperature. The analysis results show that the use of this data fusion method improved the sensor measurement accuracy, decrease the influence of abnormal data of the system, and enhances the system stability and reliability of the control system on the accuracy of the criterion.

\section{Acknowledgment}


This work was supported by the Foundation of Tianjin University of Technology and Education (Project No.KJ15-06) and the Education Reform Fund Project of Tianjin University of Technology and Education (Project No.JGY2015-06).

\section{References}

[1] Gao Shang, Chen Jingbo. Research and development of intelligent greenhouse control system. Technology Enterprises. 2015, (04): 13-14.

[2] Wen Jing and Cheng Mao. The application of multisensor data fusion technology in greenhouse control. Journal of Agricultural University of Hebei. 2015, (04): 111-115.

[3] Song Qingheng. Design of vegetable greenhouse control system based on multi-sensor data fusion. Journal of Agricultural Mechanization Research. 2015, (04): 211-214.

[4] Li Fujuan, Li Shihua and Zhao Xinyuan. Design of multi - sensor data fusion algorithm of greenhouse control system. Hubei Agricultural Sciences. 2016, (16): 4287-4289.

[5] Wang Huadong, Wang Dayu. An improved adaptive weighted fusion algorithm of wireless multi-sensor data batch estimation. Chinese Journal of Sensors and Actuators. 2015 (8): 1239-1243.

[6] Tang Yapeng. Data processing based on adaptive weighted data fusion algorithm. Computer Technology and Development. 2015 (4): 53-56.

[7] Zhang Pin, Dong Weihao, Gao Datong. An optimized bayesian estimation multi-sensor data fusion method. Chinese Journal of Sensors and Actuators. 2014 (5): 643-648. 